E0277 USE OF TRADITIONAL CHINESE MEDICINE PREPARATIONS IN OUTPATIENTS WITH CORONARY HEART DISEASE IN CHINA AND ITS INFLUENCE ON THE USE OF GUIDELINERECOMMENDED THERAPIES: RESULTS FROM THE BRIDGING THE GAP ON CHD SECONDARY PREVENTION IN CHINA (BRIG) PROJECT

doi:10.1136/hrt.2010.208967.277

Xiangyu Guo, Jun Liu, Hongjuan Li. Capital Medical University, Affiliated Beijing Anzhen Hospital

Objective To probe into the use of TCM preparations in the secondary prevention and treatment of CHD in China and investigate its influencing factors and relationship with guideline-recommended therapies.

Methods A cross-sectional survey was conducted among 2,803 CHD outpatients, a representative sample of China. Interviewers (physicians) were assigned to collect information concerning patients' medical care, previous diseases/treatments, and current medication. The use of TCM preparations and its influencing factors was analysed. Results The rate of using TCM preparations was $29.5 \%$ in 2,712 patients with complete information; the rates of using four guideline-recommended Western medication classes, aspirin, $\beta$-blocker, statins and ACEI/ARB, were 84.0, 61.9, 56.8 and $60.1 \%$, respectively. Multivariate analysis showed that the rate of using TCM preparations was higher in secondary hospitals than in tertiary hospitals and was higher in patients with a longer history of CHD and/or in patients who were not taking ACEI/ARB and statins. The rate of using TCM preparations was also higher in patients without a history of percutaneous coronary intervention (PCI). Compared with those who were not taking TCM preparations (non-TCM group), the rate of using any of the four major classes were relatively lower among patients who were taking TCM preparations (TCM group). The rate of using combined therapies was $21.8 \%$ in the TCM group and $34.8 \%$ in the non-TCM group. When other factors were adjusted, the rate of guideline-recommended therapy use in the nonTCM group was 1.7 times of that in the TCM group.

Conclusion Nearly $30 \%$ of Chinese CHD patients are taking TCM preparations to manage their heart disease, especially those with a long disease course, without a history of PCI and/or treated in secondary hospitals. The effectiveness of both TCM preparations and the four guideline-recommended drug classes requires further research.

\section{E0278 INVESTIGATION OF REPEAT CORONARY REVASCULARIZATION IN BEIJING ANZHEN HOSPITAL}

doi:10.1136/hrt.2010.208967.278

Liu Chen, Yan Hongbing. Beijing Anzhen Hospital

Objectives Although data of prevalence of prior percutaneous coronary intervention (PCI) and prior coronary artery bypass graft $(\mathrm{CABG})$ are available in the European Heart Survey Programme, there is still lack of large scale cross-sectional study in China. This survey intended to investigate current status of secondary coronary revascularization in Beijing Anzhen Hospital, which is one of the largest cardiovascular centers in China.

Methods A comprehensive review of the institution's database between January 2006 and July 2009 was conducted. Patients who received coronary revascularization were divided into PCI and CABG groups. Demographic information, concomitant diseases, peri-operative laboratory examinations, angiographic features and surgery information of consecutive patients who underwent coronary revascularization were collected.

Results A total of 20,299 patients were included in the analysis, of which $68.58 \%(13,922)$ patients received PCI and $31.42 \%(6377)$ underwent $\mathrm{CABG}$. Compared to PCI group, the mean age of CABG group was significant older $(61.8 \pm 9.4$ vs $59.7 \pm 10.8$, respectively, $\mathrm{p}<0.001$ ). The proportion of male patients in CABG group was also higher than PCI group (70.4\% vs $68.0 \%$, respectively, $\mathrm{p}=0.001)$. The prevalence of prior PCI or CABG history was 9.03\% (1257/13922) and $2.24 \%(312 / 13922)$ respectively, and $0.54 \%(75 / 13922)$ of patients had both of them. of the 6377 patients underwent the index CABG, 5.41\% (345/6377) had prior PCI, and 0.25\% (16/6377) had prior $C A B G$.

Conclusions The ratios of secondary coronary revascularization were still high in patients treated with PCI or CABG. The comprehensive control of cardiovascular risk factors is needed to strengthen.

\section{e0279 ALTERATION OF REGIONAL PULSE WAVE VELOCITY IN BEIJING GENERAL RESIDENTS AND ITS RELATIONSHIP WITH METABOLIC SYNDROME COMPONENTS}

doi:10.1136/hrt.2010.208967.279

Bian Suyan, Luo Leiming, Xiao Tiehui, Qi Liping, Wu Hongmei, Xiao Wenkai, Sheng Li, Duan Liufa. Chinese Pla General Hospital

Objective To investigate the alteration of regional pulse wave velocity (PWV) in Beijing general residents with metabolic syndrome (MS) and analyse its related factors.

Methods All the adult participants in this cross-sectional investigation were recruited from 3 big communities during their annual physical examination. Regional arterial stiffness was assessed simultaneously by measuring PWV in three arterial segments, the carotid-femoral (cFPWV), carotid-radial (crPWV) and carotid- ankle PWV (caPWV). Demographic characteristics and basal biochemical parameters including height, weight, waist and hip circumference, blood pressure, and serum levels of glucose, lipid, uric acid and creatinine were collected. MS was identified according to the criteria from the International Diabetes Federation definition.

Results A total of 2439 citizens (age ranging from 18 to 92 years) were enrolled into this study, which included 732 participants with MS (prevalence, 30.01\%). The subjects with MS were older (53.25 \pm 13.73 vs $50.03 \pm 15.73, \mathrm{p}<0.001)$, had higher cfPWV, caPWV, crPWV $(\mathrm{m} / \mathrm{s}, 11.68 \pm 2.92$ vs $10.24 \pm 2.43,9.46 \pm 1.73$ vs $8.76 \pm 2.23$, $9.76 \pm 1.52$ vs $9.42 \pm 1.45, \mathrm{p}<0.001$ for all), and increased occurrence of cardiovascular diseases $(18.3 \%$ vs $9.1 \%, \mathrm{p}<0.001)$. Partial correlation analysis after adjustment for age and sex showed that pulse pressure, LDL-C, uric acid and all component of MS were significantly related to the values of cfPWV and caPWV ( $p<0.05$ for all). In multivariate stepwise linear regression models, MS as a whole was an independent determinant for all the three regional PWV. The components of MS showed different effects on the regional PWV elevation. In detail, increased systolic blood pressure and hyperglycemia correlated with enhanced cfPWV and caPWV, while central obesity affected cFPWV only. Further, the diastolic blood pressure among the MS components independently affected crPWV, and serum triglyceride and high density lipoprotein level had little effect on PWV.

Conclusion MS contributes to the occurrence of increased arterial stiffness independently of other known cardiovascular risk factors. Among its related components, central obesity, hypertension and hyperglycemia are the critical factors determining arteriosclerosis.

\section{e0280 THE EFFORTS OF SWIMMING EXERCISE ON THE EXPRESSION OF PPAR- $\gamma$ AND LIPID METABOLISM IN THE APOE KNOCKOUT MICE}

doi:10.1136/hrt.2010.208967.280

Cai Ying, Liu Suixin, Sun Chuihua, Xie Kangling. 'Cardiac Rehabilitation Center, Xiangya Hospital of Central South University, Changsha, Hunan, China

Objectives To establish a model of ApoE knockout mice with insulin resistance induced by high fat diet, and observed the effect of 
swimming training on serum free fatty acid (FFA) and the expression of peroxisome proliferator-activated receptor (PPAR- $\gamma$ ), carnitine palmitoy1 transferase-1 (CPT-I), medium-chain acy1-coenzyme A dehydrogenase (MCAD) mRNA, to explore the mechanisms that Why swimming training could improve insulin resistance.

Methods Twenty-six male ApoE knockout mice were randomly divided into groups: the high-fat diet group (HFD, $n=13$ ) and the high-fat diet group with exercise training (HFD+Ex, $n=13)$. The HFD+Ex group were fed with high-fat diet with exercise training for 12 weeks. The treatment of HFD group was identical to the HFD+Ex group except to swimming training. And another ten healthy male C57BL/6J (ND, $\mathrm{n}=10$ ) mice as the control group were, which were fed with normal diet for 12 weeks. After 12 weeks experiment, the mice were dissected, the livers were excided off immediately. Serum insulin, glucose were determined and Homa-IRI was calculated to ascertain the establishment of insulin resistance. Serum total cholesterol (TC), trigiyceride (TG), high-density lipoprotein cholesterol (HDL), low-density lipoprotein cholesterol (LDL), free fatty acid (FFA) were determined. The PPAR- $\gamma$, CPT-I, MCAD mRNA in liver were estimated by reverse transcription PCR (RT-PCR).

Results 1. Compared with the ND group, the body weight of the HFD group was significantly higher $(\mathrm{p}<0.05)$. Compared to the HFD group, the body weight of the HFD+Ex group was significantly lower $(p<0.05)$. 2. Compared with the ND group, fasting glucose, insulin and Homa-IRI of the HFD group was significantly higher $(p<0.01)$. Compared with the HFD group, fasting insulin, glucose and HOMA-IR of the HFD+Ex group was significantly lower $(p<0.05,0.01,0.01)$. 3. Compared with the ND group, TC, LDL, FFA of HFD group was significantly higher $(p<0.01)$, TC, LDL of the HFD+Ex group was still higher $(p<0.01)$. Compared with the HFD group, TC, LDL, FFA of the HFD+Ex group was significantly lower $(p<0.05,0.05,0.01)$, HDL was significantly higher $(p<0.05)$. 4. Compared with the ND group, the expression of PPAR- $\gamma$, CPT- 1 , MCAD mRNA of the HFD group were significantly deceased $(p<0.01)$; Compared with the HFD group, the expression of PPAR$\gamma$, CPT-1, MCAD mRNA of the HFD group were significantly increased $(p<0.01)$.

Conclusion 1. High-fat diet could induce insulin resistance of ApoE Knockout mice. 2. Swimming training could improve insulin resistance of ApoE knockout mice. 3. Swimming training could improve insulin resistance possibly through upregulating the expression of PPAR- $\gamma$, CPT-1, MCAD mRNA.

Funding This study is supported by Natural Sciences Foundation of Hunan. N0.06SK3090.

\section{e0281 RELATIONSHIP BETWEEN ALBUMINURIA AND BLOOD PRESSURE LEVEL IN PATIENTS WITH ESSENTIAL HYPERTENSION}

doi:10.1136/hrt.2010.208967.281

\begin{abstract}
${ }^{1}$ Sun Ningling, ${ }^{1}$ Wang Hongyi, ${ }^{2}$ Zhu Dingliang, ${ }^{3}$ Liao Yuhua, ${ }^{4}$ Lin Shuguang, ${ }^{5}$ Chen Xiaoping. ${ }^{1}$ People's Hospital Peking Universtiy; ${ }^{2}$ Hypertension Research Institute Shanghai; ${ }^{3}$ Union Hospital Wuhan; ${ }^{4}$ Guangdong General Hospital; ${ }^{5}$ Sichun University Westchina Hospital
\end{abstract}

Objective To investigate the relationship between albuminuria detection rate and blood pressure level or body weight index (BMI) in patients with essential hypertension in China's five regions.

Method 5021 patients with clearly diagnosed essential hypertension but without diabetes were enrolled in our study. The participants came from five cities of China. Urinary albumin/creatinine ratio was measured in these patients for two times. The relationships between albuminuria and levels of blood pressure and BMI were analysed.

Results 1 . There wasn't any difference of the albuminuria incidence between patients less than 60-year-old and equal to or more than
60 -year-old. The longer the hypertension exists, the higher the proportion of albuminuria. 2. The incidence of albuminuria associated with blood pressure levels significantly. The higher the blood pressure (BP) level, the more the urine protein excretion. The albuminuria detection rate in patients with normal blood pressure, high normal blood pressure, I, II or III stage hypertension were $26.3 \%$, $27.3 \%, 28.7 \%, 31.5 \%$ and $40.3 \%$ respectively. 3 . The incidence of albuminuria was different in patients with uncontrolled BP (BP $>=140 / 90 \mathrm{~mm} \mathrm{Hg}$ ) compared with those with well controlled $\mathrm{BP}(<140 / 90 \mathrm{~mm} \mathrm{Hg})(27.1 \%$ vs $30.2 \%, \mathrm{p}<0.05)$. 4. The incidence of albuminuria was in obese patients compared with those with normal body weight at equal blood pressure level, but the difference wasn't statistically significant. 5. Patients with albuminuria had more heart, cerebral or renal events than whom without proteinuria. Conclusion The detection rate of proteinuria in hypertensive patients without known diabetes was $28.8 \%$ in China, among them the incidence of microalbuminuria was $18.6 \%$, and the incidence of clinical albuminuria was $10.2 \%$. Uncontrolled BP was important risk factor of proteinuria.

\section{E0282 EFFECT OF COMPREHENSIVE INTERVENTION ON ANXIETY AND DEPRESSIVE SYMPTOMS IN PATIENTS AFTER ACS}

doi:10.1136/hrt.2010.208967.282

${ }^{1}$ Liu Guanghui, ${ }^{1}$ Wu Xianzheng, ${ }^{2}$ Ma Wenlin, ${ }^{1}$ Su Lijie, ${ }^{3} \mathrm{Hu}$ Dayi. ${ }^{1}$ Emergency Internal Medicine, Tongji Hospital, Tongji University; ${ }^{2}$ Department of Cardiology, Tongji Hospital, Tongji University; ${ }^{3}$ Heart Center Peking University Peoples Hospital

Objective To evaluate the effect of comprehensive intervention to the prognosis of depressive and/or anxiety symptoms in future. To provide a reasonable and reliable intervention instrument to treat ACS patients.

Methods To select acute coronary syndrome with anxious and/or depressive symptoms patients 268 examples, all patients were divided into the intervention group 134 examples and the control group 134 examples. On the base of conventional therapy, Patients of intervention group received comprehensive intervention (health education, exercise, psychological relaxation et al). Before the intervention and after follow-up test the HADS, to follow up with the patients to observe the dynamic change of emotion. The state of anxiety and depression in all patients were analysed according to psychological test scale.

Results 1. Total 256 example patient include the intervention group $(n=130)$ and the control group $(n=126)$, after giving the treatment has carried on at least two times evaluation. Because loses the revisit 6 examples, died 4 examples, uncompliant patients 6 examples, completed finally experiments 240 examples. 2. Comparing two group's emotion in the two group patients respectively at baseline and after intervention. The HADS scores carried on the difference examination separately: Before the comprehensive intervention, the intervention group's HAD-a, HAD-d, HAD-t are 8.92 $\pm 3.72,8.73 \pm 3.41,17.68 \pm 3.72$ respectively, the control group's scores are 8.67 $\pm 3.13,8.52 \pm 3.06,17.47 \pm 3.27$ respectively, $\mathrm{p}>0.05$; After intervention, for the invention group, HAD-a, HAD-d, HAD-t are 5.82 $\pm 1.41,6.84 \pm 1.86,11.93 \pm 1.59$; The control group's are $6.76 \pm 1.31,7.28 \pm 1.58,13.62 \pm 1.36$ respectively, $\mathrm{p}<0.05$.

Conclusions For the intervention group patients, comprehensive intervention (Coronary heart disease treatment, Health education, psychological counseling, Relaxation training, taking exercise et al) may obviously alleviate the anxiety and/or depressive symptoms in ACS patients, it is useful to the prognosis improvement of QOL. 2. On the basis of medication for ACS, for the control group patients, the anxiety and/or depressive symptom has been alleviated to some extent. 